BMJ Open

Diabetes

Research

\& Care

\title{
Association between serum fibroblast growth factor 21 level and sight- threatening diabetic retinopathy in Chinese patients with type 2 diabetes
}

\author{
Shi Jin, ${ }^{1}$ Ning $\mathrm{Xia}^{2}{ }^{2}$ Lingling Han (D) ${ }^{1}$
}

To cite: Jin S, Xia N, Han L. Association between serum fibroblast growth factor 21 level and sight-threatening diabetic retinopathy in Chinese patients with type 2 diabetes. BMJ Open Diab Res Care 2021;9:e002126. doi:10.1136/ bmjdrc-2021-002126

NX and LH contributed equally.

Received 11 January 2021 Revised 25 February 2021 Accepted 6 March 2021

\section{Check for updates}

C) Author(s) (or their employer(s)) 2021. Re-use permitted under CC BY-NC. No commercial re-use. See rights and permissions. Published by BMJ.

${ }^{1}$ Department of Endocrinology, the Fourth Affiliated Hospital of China Medical University, Shenyang, China

${ }^{2}$ Department of Ophthalmology, the Fourth People's Hospital of Shenyang, Shenyang, China

Correspondence to Dr Lingling Han; hanll_1971@126.com and Dr Ning Xia;

16186979@qq.com

\section{ABSTRACT}

Introduction We conducted this cross-sectional study to explore the relationship between serum fibroblast growth factor 21 (FGF21) level and sight-threatening diabetic retinopathy (STDR).

Research design and methods $A$ total of 654 patients with type 2 diabetes were recruited. Diabetic retinopathy (DR) was evaluated by the bilateral retinal photography, and patients were assigned into groups of no DR (NDR) $(\mathrm{n}=345,52.75 \%)$, non-sight-threatening diabetic retinopathy (NSTDR) $(n=207,31.65 \%)$, involving patients with mild or moderate non-proliferative retinopathy (NPDR) and STDR ( $n=102,15.60 \%)$, including those with severe NPDR or proliferative diabetic retinopathy (PDR). Serum FGF21 levels were quantified by a sandwich ELISA. Patients were divided into quartiles according to their serum FGF21 level.

Results There was a significant difference in serum FGF21 level among the three groups of patients $(p<0.01)$. Compared with other quartiles (Q1-Q3), the patients in Q4 had a higher prevalence of DR and STDR $(p<0.05)$. Compared with Q1, a positive association was observed between serum FGF21 level and DR in Q3 and Q4 $(p<0.01)$. After adjusting for age, gender and other risk factors, serum FGF21 level in Q4 was found to be associated with increased risk of DR and STDR $(p<0.01)$. Serum FGF21 level was noted as an independent risk factor for DR and STDR $(p<0.01)$. Serum FGF21 level $>478.76 \mathrm{pg} / \mathrm{mL}$ suggested the occurrence of DR and that level $>554.69 \mathrm{pg} / \mathrm{mL}$ indicated STDR $(\mathrm{p}<0.01)$.

Conclusions Serum FGF21 level was a biomarker for the risk of developing DR or STDR. The risk of STDR increased when the serum FGF21 level of patients with type 2 diabetes was $>554.69 \mathrm{pg} / \mathrm{mL}$.

\section{INTRODUCTION}

With the improvement of people's living standards and the change of their lifestyle, diabetes has become one of the most important chronic non-communicable diseases threatening the global health. According to the latest statistics released in 2019 by the International Diabetes Federation, it is estimated that 463 million people have diabetes and this number is projected to reach 578 million by 2030 , and 700 million by

\section{Significance of this study}

What is already known about this subject?

- An association between serum fibroblast growth factor 21 (FGF21) and obesity, diabetes or atherosclerosis has been reported in previous studies; however, the effect of elevated serum FGF21 level on the occurrence of diabetic retinopathy (DR) was unclear.

What are the new findings?

- In this research, we found patients with diabetes with $\mathrm{DR}$, especially sight-threatening diabetic retinopathy (STDR) had significantly higher serum levels of FGF21 than those without DR.

- Meantime, with the increase of serum FGF21 level, the prevalence of DR and STDR increased gradually amongpatients with type 2 diabetes, and the serum FGF21 level $>554.69 \mathrm{pg} / \mathrm{mL}$ indicates over eighttime enhancement of STDR occurrence.

How might these results change the focus of research or clinical practice?

- Our study first reported the close relationship between elevated serum FGF21 level and STDR, providing an idea in STDR screening and a pathway for DR therapy in future.

2045. Diabetes is becoming one of the fastest growing global health challenges in the 21st century and has reached alarming levels; at present, nearly half a billion people are living with diabetes worldwide. In 2019, China included the largest number of adults with diabetes aged $20-79$ years, and this trend is predicted to remain so in 2030 and 2045 . $^{1}$

Diabetic retinopathy (DR) is one of the most common microvascular complications of diabetes and has become the leading cause of new blindness in adults aged 20-74 years. ${ }^{2}$ It was estimated that $23 \%$ of Chinese patients with diabetes have retinal complications and there was a lack of effective DR screening methods in inland areas. ${ }^{3} \mathrm{DR}$ is characterized 
by blurred vision, vision loss and even blindness. Several studies have shown that the duration of diabetes, blood glucose control level and blood pressure are major independent risk factors for the occurrence and development of DR. ${ }^{4}$ It was recommended that patients with type 2 diabetes should receive comprehensive eye examination immediately after diagnosis, and the frequency of review should not be less than once every 2 years. The frequency of examination should be increased if there is any progress of retinopathy or vision loss. ${ }^{5}$ Because of the shortage of obvious symptoms or signs of early DR and the lack of sufficient equipment or ophthalmologists, there was a great difficulty to early diagnosis and early treatment of DR. Therefore, finding a more effective, convenient and economical means of screening DR has become an urgent medical and health problem that significantly requires scholars' attention.

Fibroblast growth factor 21 (FGF21) is a polypeptide with 210 amino acids, which is preferentially produced in the liver and plays a significant role in atherosclerosis formation, regulating glucose and lipid metabolism and insulin sensitivity. ${ }^{6}$ Several epidemiological studies reported the increased serum level of FGF21 in patients with obesity, diabetes and metabolic syndrome. ${ }^{7}$ Meanwhile, its association with cardiovascular events has received increasing attention. It was reported that FGF21 was closely associated with acute myocardial infarction and lower extremity atherosclerotic disease. ${ }^{89}$ However, a limited number of studies have discussed the association between FGF21 and DR. The current research was conducted to assess the difference in the serum FGF21 level in patients with and without sight-threatening diabetic retinopathy (STDR), and to evaluate the value of this serum marker for screening STDR in Chinese patients with type 2 diabetes.

\section{METHODS}

\section{Study population}

A number of 654 outpatients admitted to the Fourth Affiliated Hospital of China Medical University from January 2018 to October 2019 were included in this study, who were diagnosed with type 2 diabetes mellitus (T2DM) according to the 1999 WHO criteria and the 2012 American Diabetes Association standards of medical care in diabetes. ${ }^{10}$ Those patients with type 1 diabetes or specific types of diabetes, acute complications of diabetes, fundus lesions owing to orbital tumor, trauma, malignant hypertension or other definite causes and secondary nephropathy caused by acute glomerulonephritis, pyelonephritis, nephrolithiasis and so on were excluded. In total, 347 males $(56.88 \%)$ and 307 females $(53.06 \%)$ were enrolled with a mean age of $57.90 \pm 11.05$ years and the mean duration of diabetes was $8.74 \pm 6.25$ years.

\section{Data collection and anthropometric measurement}

Data related to the patients' sex, age, duration of diabetes and history of smoking, drinking, coronary artery disease and cerebral apoplexy were collected. A patient's height was measured to the nearest centimeter. Weight was determined with a traditional spring balance that was kept on a firm horizontal surface. The body mass index (BMI) was calculated as the weight in kilograms divided by height in meters squared. Blood pressure was measured by the same nurse using a mercury sphygmomanometer for three times and then averaged.

\section{Laboratory measurements}

Blood samples were collected from an antecubital vein for measuring the levels of total cholesterol, triglyceride (TG), high-density lipoprotein cholesterol (HDL-C), low-density lipoprotein cholesterol, blood urea nitrogen (BUN), serum creatinine $(\mathrm{Cr})$, uric acid, fasting plasma glucose and 2-hour postprandial blood glucose by an enzymatic method with an automatic biochemical analyzer (Cobas c701; Roche, Basel, Switzerland). Glycosylated hemoglobin (HbAlc) level was measured by high-pressure liquid chromatography using the Variant II machine (Bio-Rad Laboratories, Hercules, California, USA). Glycosylated albumin (GA) level was determined by the liquid enzymatic assay using the Glamour 2000 automatic biochemical analyzer (MD, Silicon Valley, California, USA). The serum FGF21 level was determined by ELISA (ab222506, Abcam, Shanghai, China). The intra-assay and inter-assay variations were $7.8 \%$ and $9.1 \%$, respectively. Continuous dilution of recombinant FGF21 was used as the standard. Repeated measurements were carried out for all samples. All the patients were divided into quartiles according to their serum FGF21 level (pg/ $\mathrm{mL}$ ) as follows: quartile 1 (Q1, FGF21 <388), quartile 2 (Q2, $388 \leqq$ FGF21 <455), quartile 3 (Q3, $455 \leqq$ FGF21 $<579)$ and quartile $4(\mathrm{Q} 4, \mathrm{FGF} 21 \geqq 580)$.

\section{Ophthalmic examination and grading of DR}

All participants received eye examinations by the same experienced ophthalmologist and fundus photography was performed using a 45-degree 6.3-megapixel digital non-mydriatic camera (Canon CR-45NM, Lake Success, New York, USA). The photographs were graded according to the International Classification of Diabetic Retinopathy scale ${ }^{11}$ : (i) no changes in DR; (ii) mild nonproliferative diabetic retinopathy (NPDR); (iii) moderate NPDR; (iv) severe NPDR and (v) proliferative diabetic retinopathy (PDR). The degree of DR was determined according to the grading in the worse eye. Then, the patients were divided into three groups: no DR (NDR) $(\mathrm{n}=345,52.75 \%)$, non-sight-threatening diabetic retinopathy (NSTDR) (n=207, 31.65\%), involving patients with mild or moderate NPDR and STDR $(n=102,15.60 \%)$, including those with severe NPDR or PDR.

\section{Statistical analysis}

SPSS V.16.0 software (IBM, Armonk, New York, USA) was used to carry out statistical analysis. Data were expressed as mean $\pm \mathrm{SD}$ for continuous variables and percentages (\%) for categorical variables. Differences among the 
groups were detected using analysis of variance test or Kruskal-Wallis test as appropriate for measurement data, as well as the $\chi^{2}$ test for categorical data. Multivariable logistic regression analysis was performed to evaluate the OR and associated factors. We calculated the OR with 95\% CI of DR associated with FGF21 in three logistic regression models: a non-adjusted model; age-adjusted, sex-adjusted model and a multivariable regression model adjusted for all variables $(\mathrm{p}<0.05)$. After the analysis of correlation, receiver operating characteristic (ROC) curve was used to find the cut-point of FGF21 to predict DR and STDR. Two-tailed $\mathrm{p}<0.05$ was considered statistically significant.

\section{RESULTS}

\section{Comparing the patients' clinical characteristics}

Between January 2018 and October 2019, a total of 654 patients with type 2 diabetes were enrolled in this study and all the patients were divided into three groups according to the results of fundus examination, including NDR $(\mathrm{n}=345,52.75 \%)$, NSTDR $(\mathrm{n}=207,31.76 \%)$ and STDR $(\mathrm{n}=102,15.60 \%)$. Overall, the study population (347 males and 307 females) had a mean age of $57.90 \pm 11.05$ years, the duration of T2DM $8.74 \pm 6.25$ years and the median serum level of FGF21 was $450.50 \pm 101.46 \mathrm{pg} / \mathrm{mL}$, with an IQR of $190.05-545.55 \mathrm{pg} / \mathrm{mL}$.

The patients' demographic and clinical characteristics are summarized in table 1 . There were significant differences in duration of diabetes, blood glucose level, renal function and serum FGF21 level among the three groups of patients. Compared with patients in NDR group, those in NSTDR and STDR groups had significantly longer duration of diabetes and higher serum levels of HbA1c, GA, Cr and FGF21 (572.7 $\pm 79.5,625.8 \pm 83.7$ vs $326.8 \pm 81.6 \mathrm{pg} / \mathrm{mL}$ ). Besides, compared with patients in NSTDR group, those in STDR group had remarkably longer duration of diabetes and higher serum levels of BUN, Cr and FGF21 (625.8 \pm 83.7 vs $572.7 \pm 79.5 \mathrm{pg} / \mathrm{mL})$.

\section{Association of serum FGF21 level with DR and STDR}

In order to compare prevalence of DR and investigate association between DR and serum FGF21 level, we also divided all the patients into groups of Q1-Q4 according to their serum FGF21 level. As shown in figure 1, with the increase of serum FGF21 level, the prevalence of DR and STDR increased gradually. Compared with Q1

\begin{tabular}{|c|c|c|c|c|}
\hline Characteristics & NDR & NSTDR & STDR & $P$ value \\
\hline Case (male/female) & $345(185 / 160)$ & $207(107 / 100)$ & $102(55 / 47)$ & / \\
\hline Duration of diabetes (year) & $7.11 \pm 5.76$ & $9.95 \pm 6.24^{*}$ & $11.81 \pm 7.27^{\star} \dagger$ & 0.000 \\
\hline $\mathrm{BMI}\left(\mathrm{kg} / \mathrm{m}^{2}\right)$ & $25.28 \pm 3.64$ & $25.31 \pm 3.14$ & $25.11 \pm 3.74$ & 0.679 \\
\hline $\mathrm{DBP}(\mathrm{mm} \mathrm{Hg})$ & $78.84 \pm 7.82$ & $77.59 \pm 7.31$ & $78.61 \pm 7.97$ & 0.678 \\
\hline Smoking (\%) & 31.2 & 36.4 & 26.0 & 0.226 \\
\hline Drinking (\%) & 17.4 & 19.2 & 15.4 & 0.423 \\
\hline Coronary artery disease (\%) & 8.7 & 10.2 & 14.9 & 0.241 \\
\hline Cerebral infraction (\%) & 6.2 & 9.6 & 8.1 & 0.277 \\
\hline GA (\%) & $20.47 \pm 6.32$ & $22.18 \pm 5.74^{*}$ & $22.79 \pm 6.49^{\star}$ & 0.000 \\
\hline BUN (mmol/L) & $5.24 \pm 1.29$ & $5.31 \pm 1.15$ & $6.03 \pm 2.02^{*} \dagger$ & 0.008 \\
\hline $\mathrm{Cr}(\mu \mathrm{mol} / \mathrm{L})$ & $62.44 \pm 15.81$ & $65.56 \pm 15.28^{\star}$ & $71.55 \pm 20.84^{\star} \dagger$ & 0.001 \\
\hline $\mathrm{UA}(\mu \mathrm{mol} / \mathrm{L})$ & $315.58 \pm 82.92$ & $306.27 \pm 79.05$ & $332.24 \pm 84.47$ & 0.071 \\
\hline TC (mmol/L) & $4.92 \pm 1.05$ & $4.80 \pm 1.03$ & $5.11 \pm 1.24$ & 0.210 \\
\hline $\mathrm{TG}(\mathrm{mmol} / \mathrm{L})$ & $1.79 \pm 1.46$ & $1.99 \pm 1.51$ & $1.81 \pm 1.50$ & 0.140 \\
\hline $\mathrm{HDL}-\mathrm{C}(\mathrm{mmol} / \mathrm{L})$ & $1.12 \pm 0.27$ & $1.08 \pm 0.25$ & $1.15 \pm 0.32$ & 0.100 \\
\hline LDL-C (mmol/L) & $2.95 \pm 0.86$ & $2.83 \pm 0.79$ & $3.02 \pm 0.77$ & 0.177 \\
\hline FGF21 (pg/mL) & $326.8 \pm 81.6$ & $572.7 \pm 79.5^{\star}$ & $625.8 \pm 83.7^{\star} \dagger$ & 0.000 \\
\hline
\end{tabular}

${ }^{*} \mathrm{P}<0.01$ vs NDR.

$\dagger \mathrm{P}<0.01$ vs NSTDR.

BMI, body mass index; BUN, blood urea nitrogen; Cr, creatinine; DBP, diastolic blood pressure; FGF21, fibroblast growth factor 21; FPG, fasting plasma glucose; GA, glycosylated albumin; HbA1c, glycosylated hemoglobin; HDL-C, high-density lipoprotein cholesterol; LDL-C, low-density lipoprotein cholesterol; NDR, no diabetic retinopathy; NSTDR, non-sight threatening diabetic retinopathy; PPG, postprandial blood glucose; SBP, systolic blood pressure; STDR, sight-threatening diabetic retinopathy; TC, total cholesterol; TG, triglyceride; UA, uric acid. 


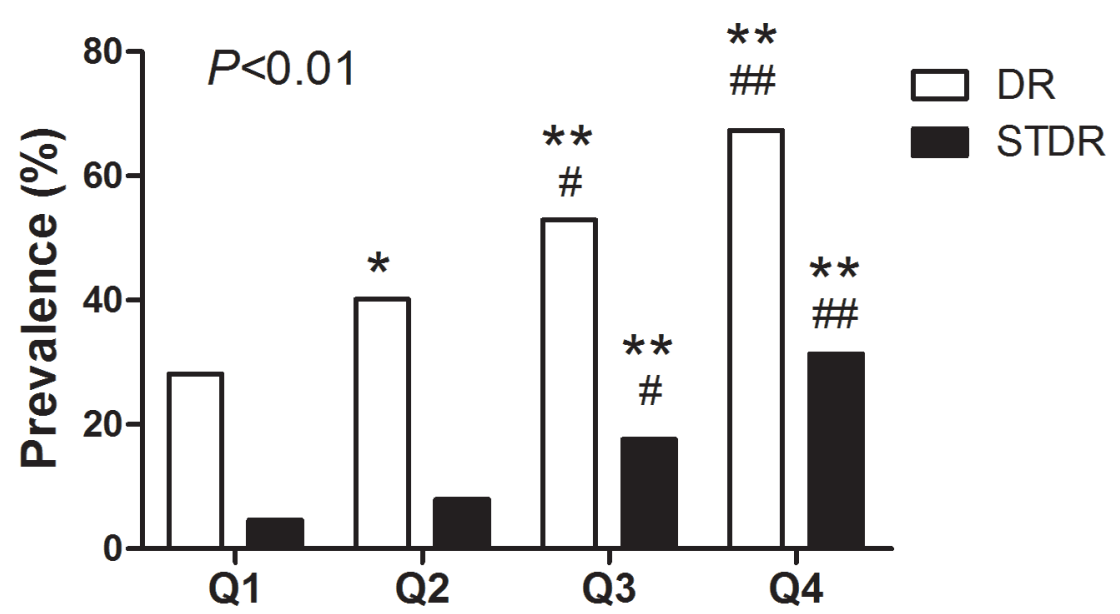

Figure 1 Prevalence of DR and STDR among groups of Q1-Q4. * $P<0.05,{ }^{\star *} p<0.01$ vs $Q 1 ; \# p<0.05$, \#\#p<0.01 vs Q2. DR, diabetic retinopathy; STDR, sight-threatening diabetic retinopathy.

and Q2 groups, the patients in Q3 and Q4 groups had significantly higher prevalence of DR and STDR (DR prevalence (\%,Q1-Q4): 28.15, 40.17, 52.94 and 67.36, respectively; STDR prevalence $(\%, \mathrm{Q} 1-\mathrm{Q} 4): 4.62,7.95$, 17.65 and 31.38, respectively; all $\mathrm{p}<0.05)$. Table 2 shows the association between serum FGF21 level and DR severity. Compared with $\mathrm{Q} 1$ group, a significant positive association was clearly observed between serum FGF21 level and DR in Q3 group (OR 4.63; 95\% CI 1.09 to 6.44) and Q4 (OR 6.98; 95\% CI 1.98 to 11.50) in non-adjusted model. In the age-adjusted, sex-adjusted model and the multivariable regression model, the significant association could be observed (all $\mathrm{p}<0.05$ ). The similar results appeared in models of STDR. The positive association between serum FGF21 level and STDR was significant in Q3 and Q4 groups compared with Q1 group (reference) in the three models (all $\mathrm{p}<0.05$ ). Besides, the association between serum FGF21 level and STDR was stronger than that of DR. Compared with Q1 group, the multivariable OR $(95 \% \mathrm{CI})$ of STDR in Q4 group was 10.22 (5.25 to 15.06 ) and 9.10 (5.70 to 15.79) in the age-adjusted, sexadjusted model and the multivariable regression model, respectively (both $\mathrm{p}<0.01$ ).

\section{Associated factors for DR}

Using multivariate logistic regression analysis, the independent risk factors for DR included serum FGF21 level $(\beta=0.73)$, age $(\beta=0.05)$, duration of diabetes $(\beta=0.05)$ and HDL-C level $(\beta=0.02)($ all $\mathrm{p}<0.05)$. Meanwhile, the independent risk factors for STDR were serum FGF21 level $(\beta=0.89)$, age $(\beta=0.12)$, duration of diabetes $(\beta=0.04)$ and diastolic blood pressure $(\beta=0.02) \quad($ all $p<0.05$; table 3$)$.

\section{The predictive value of serum FGF21 level for DR and STDR}

Regarding the close relationship between serum FGF21 level and severity of DR, especially STDR, ROC analysis was performed and detected the optimal cut-off point of serum FGF21 level in hinting DR and STDR. As illustrated in figure 2A, in DR, the optimal cut-off point of serum FGF21 level was $478.76 \mathrm{pg} / \mathrm{mL}$. The Youden Index at this level was 0.19 ; besides, its sensitivity and specificity were
$42.4 \%$ and $76.2 \%$, respectively. The $\chi^{2}$ test showed that the OR was 9.72 (95\% CI 4.659 to 12.851) for detecting the presence of DR $(\mathrm{p}<0.01)$. It was revealed that the optimal cut-off point of serum FGF21 level for STDR was $554.69 \mathrm{pg} / \mathrm{mL}$ (figure 2B). The Youden Index at this level was 0.21 ; in addition, its sensitivity and specificity were $67.3 \%$ and $84.6 \%$, respectively. The $\chi^{2}$ test showed that the OR was 8.55 (95\% CI 4.589 to 12.792) for detecting the presence of STDR $(\mathrm{p}<0.01)$.

\section{DISCUSSION}

In the present study, we aimed at investigating the relationship between serum FGF21 level and DR, as well as evaluating the predictive value of serum FGF21 level for STDR in patients with type 2 diabetes. The results revealed that serum FGF21 level was significantly associated with the severity of DR and serum FGF21 level $>554.69 \mathrm{pg} / \mathrm{mL}$ predicted over eight-times elevated risk of the presence of STDR. This suggests that serum FGF21 level might be a potential indicator of the progression of severe DR in Chinese patients with type 2 diabetes.

Differences in serum FGF21 level between healthy volunteers and patients with type 2 diabetes were first observed in patients with abnormal glucose level and lipid metabolism. The baseline serum FGF21 levels were found significantly higher in patients with obesity, diabetes and metabolic syndrome than those in healthy subjects. ${ }^{712} 13$ A community-based study recruited 232 Chinese patients and found that overweight individuals or individuals with obesity had significantly higher plasma FGF21 levels than normal-weight individuals, and plasma FGF21 level was significantly associated with a number of indicators, such as BMI, waist circumference, waist-to-hip ratio and body fat content. ${ }^{12}$ A follow-up study of 1900 adults reported a remarkable increase in serum FGF21 levels in patients with type 2 diabetes and prediabetes. ${ }^{13}$ Gao et al demonstrated that higher serum FGF21 levels were positively associated with metabolic syndrome in patients with T2DM and significantly positively correlated with body 


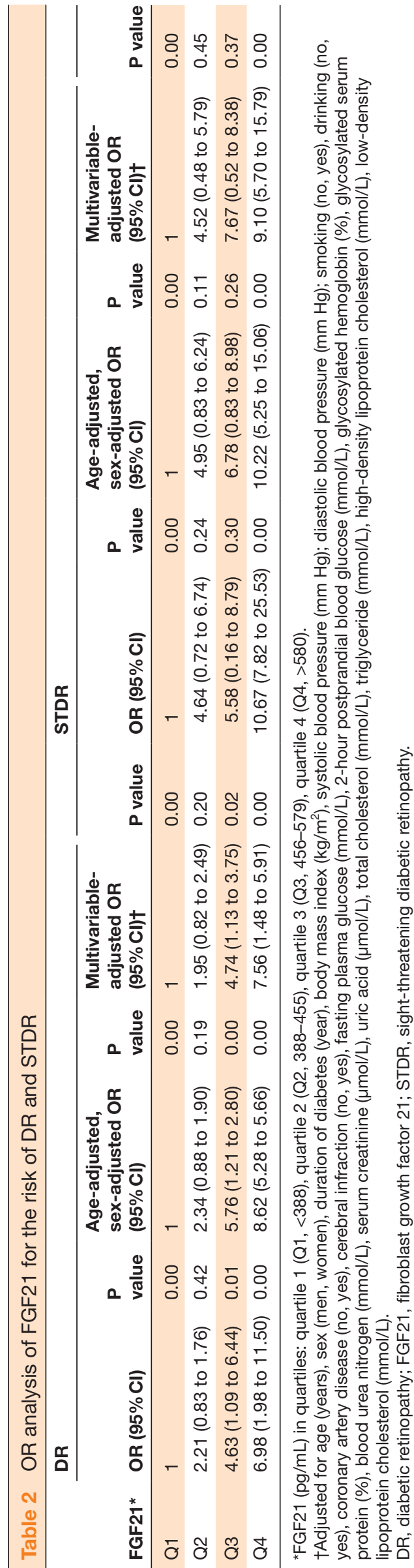

Table 3 Independent factors with DR and STDR by multiple logistical regression

\begin{tabular}{|c|c|c|c|c|}
\hline & & SE & Wald & $P$ value \\
\hline \multicolumn{5}{|l|}{ DR } \\
\hline FGF21 & 0.73 & 0.29 & 7.66 & $<0.01$ \\
\hline Age & 0.05 & 0.02 & 12.07 & $<0.01$ \\
\hline Diabetes duration & 0.05 & 0.02 & 4.03 & 0.04 \\
\hline $\begin{array}{l}\text { High-density } \\
\text { lipoprotein cholesterol }\end{array}$ & 0.02 & 0.01 & 2.66 & 0.05 \\
\hline \multicolumn{5}{|l|}{ STDR } \\
\hline FGF21 & 0.89 & 0.34 & 8.79 & $<0.01$ \\
\hline Age & 0.12 & 0.25 & 8.24 & $<0.01$ \\
\hline Diabetes duration & 0.04 & 0.02 & 4.74 & 0.04 \\
\hline $\begin{array}{l}\text { Diastolic blood } \\
\text { pressure }\end{array}$ & 0.02 & 0.01 & 2.78 & 0.04 \\
\hline
\end{tabular}

DR, diabetic retinopathy; FGF21, fibroblast growth factor 21; STDR, sight-threatening diabetic retinopathy.

fat content and TG level in these subjects. ${ }^{7}$ In addition, serum FGF21 level was previously found to be strongly associated with age, insulin resistance and adverse lipid profiles. ${ }^{14}$ In the present study, we recruited 654 patients with type 2 diabetes and divided them into three groups according to the results of fundus examination, including NDR, NSTD and STDR. Overall, the median serum level of FGF21 was $449.47 \pm 101.46 \mathrm{pg} / \mathrm{mL}$, which was consistent with previous findings and even higher than that in healthy controls. ${ }^{13}$ Meanwhile, there was a significant difference in serum FGF21 level among patients with or without DR. The serum FGF21 level was consistent with the degree of DR.

Numerous epidemiological studies previously reported that age, duration of diabetes, hyperglycemia, hypertension, hyperlipidemia were summarized as the traditional risk factors for DR, ${ }^{15} 16$ which was consistent with results of the present research. In our research, serum FGF21 level was the independent risk factors for DR and STDR. And the prevalence of DR and STDR increased gradually with the increased serum FGF21 level. This close relationship between serum FGF21 level and DR was also reported by other researches. A cross-sectional study recurred 117 patients with diabetes and 68 healthy controls. It was revealed that serum FGF21 concentrations in patients with PDR or NPDR were significantly higher than those in patients without DR. ${ }^{17}$ Iranian scholars evaluated serum FGF21 level in 61 subjects (14 healthy controls, 22 diabetics without DR and 25 diabetics with DR), and found that there was a significant difference in serum FGF21 level among those subjects, however, the difference between patients with and without DR was not statistically significant. ${ }^{18}$ Another study conducted in Iran compared the serum FGF21 level between cases with and without pterygium, and observed that the mean serum FGF21 level in patients with pterygium was significantly lower than that in healthy subjects. ${ }^{19}$ In the present 


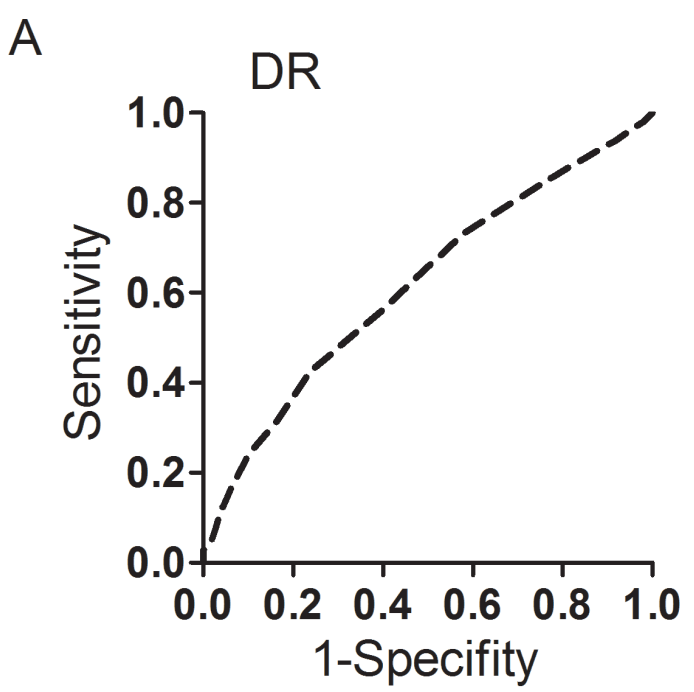

B

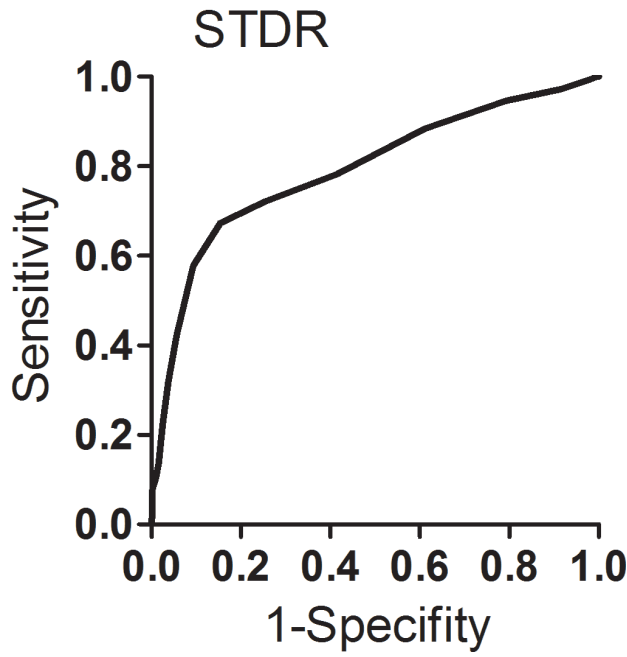

Figure 2 Receiver operating characteristic curve analysis of fibroblast growth factor-21 (FGF21) for (A) diabetic retinopathy, area under the curve $(A \cup C)=0.617(p=0.001), 95 \% \mathrm{Cl} 0.581$ to 0.652 . The optimal cut-off point of serum FGF21 level was $478.76 \mathrm{pg} / \mathrm{mL}$ (Youden Index, 0.19; sensitivity, 42.4\%; specificity, 76.2\%). (B) Sight-threatening diabetic retinopathy, $\mathrm{AUC}=0.791$ ( $\mathrm{p}=0.001), 95 \% \mathrm{Cl} 0.746$ to 0.836 . The optimal cut-off point of serum FGF21 level was $554.69 \mathrm{pg} / \mathrm{mL}$ (Youden Index, 0.21 ; sensitivity, $67.3 \%$; specificity, $84.6 \%$ ).

research, compared with the patients in Q1 group (reference), patients in Q4 group had significantly higher risks of DR and STDR after adjusting for all the associated factors. ROC analysis further revealed that when the serum FGF21 level was $>554.69 \mathrm{pg} / \mathrm{mL}$, the patients with type 2 diabetes had over eight-time increased risk of STDR

Several researches have concentrated on the association between serum FGF21 level and DR, while further study needs to be conducted due to controversial outcomes. The Fen fibrate Intervention and Event Lowering in Diabetes study that recruited 9697 participants showed that higher serum FGF21 level was associated with a high risk of baseline total microvascular disease. However, when those with microvascular disease were separately studied, serum FGF21 level was only correlated to nephropathy, and it had no association with retinopathy or other microvascular diseases. ${ }^{20} \mathrm{~A}$ number of scholars further studied the relationship between serum FGF21 level and DR, and found that cases with DR had a significantly higher serum FGF21 level than those without DR. ${ }^{17} 21$ Jung et al reported a different U-shaped relationship between serum FGF21 level and prevalence of DR. ${ }^{22}$ This discrepancy might result from the difference in patients' characteristics, such as age, blood glucose level, lipoprotein level and so on. Numerous researches pointed out that the mentioned discrepancy might be related to a relatively small sample size. The strength of our research was a larger population (654 patients) and first reported the relationship between serum FGF21 level and a subtype of DR, namely STDR.

FGF21 is a new endocrine hormone mainly produced by liver and adipose tissue, and its expression is regulated by peroxisome proliferator-activated receptor (PPAR) $\alpha$ and PPAR $\gamma$, respectively. FGF21 could be secreted into blood and has physiological functions, mainly includeing increasing the production of gluconeogenesis and ketone bodies under starvation and increasing insulin sensitivity in the postprandial state. The research focused on mechanism behind the association between serum FGF21 level and DR was relatively infrequent. However, based on the previous research related to FGF21, we could speculate the mechanism possible for the elevated serum FGF21 level with DR might include a compensatory mechanism to counteract metabolic stress and vascular endothelial dysfunction. ${ }^{23}{ }^{24}$ It was revealed that there existed FGF21 resistance in obesity or cardiovascular failure. ${ }^{25}{ }^{26}$ The term 'FGF21 resistance' was first used to describe increased circulating FGF21 levels concomitant to decreased FGF21 receptor complex expression in white adipose tissue of mice with obesity. Previous studies found that in insulin-resistant mice, the expression of FGF21 in liver and adipose tissue is increased, while the expression of FGF21 receptor is decreased, suggesting the existence of FGF21 resistance. ${ }^{23}$ Nowadays, several lines of evidence showed that the vascular system might be a target for FGF21. For example, FGF21 protects against atherosclerosis by inducing adiponectin to inhibit neointima formation and macrophage inflammation in blood vessels and suppresses hepatic cholesterol synthesis to attenuate hypercholesterolemia. ${ }^{27}$ FGF21 also acts adipocytes and renal cells to promote metabolism of angiotensin II and to mitigate hypertension and vessel injury. ${ }^{28}$ Lei Ying et al reported that FGF21 improves dilation of aorta in both type 1 diabetes and type 2 diabetes mice, probably via CaMKK2/AMPKo-mediated suppression of oxidative stress and activation of endothelial nitric oxide synthase. ${ }^{29}$ Based on these findings, we propose that the mechanism of increased FGF21 levels in DR is similar to 
those observed in hyperglycemia-associated resistance to adiponectin. In response to endothelial dysfunction, serum FGF21 might be compensatorily increased to repair microvascular legions involved in retinopathy.

According to the 2012 American Diabetes Association standards of medical care in diabetes, timely laser photocoagulation therapy based on high-quality fundus photographs can be used to reduce the risk of vision loss in patients with high-risk DR. Hence, ADA repeatedly emphasized that less-frequent eye examinations (every 2-3 years) should be performed by an ophthalmologist who is expert in diagnosing the presence of DR. To meet this demand, the use of retinal photography in remote reading by experts is recommended in areas where advanced medical equipment or qualified eye care professionals are not existed. However, the measurement of FGF21 in serum samples is efficient, common and easy to be expanded and popularized in the community hospitals. Therefore, the FGF21 assay can benefit more populations and prompt the necessity to take further eye examinations and therapies. What is important is the fact that the close relationship between serum FGF21 level and DR provided a new pathway for the therapy of DR in the future.

\section{Limitations}

One of the limitations of the present study was that no cause-and-effect relationship between serum FGF21 level and DR or STDR could be drawn due to cross-sectional nature of this study. Second, the sample size was relatively small, especially the number of cases with STDR. Moreover, this was a single-center study, which limited its clinical efficacy. A longitudinal, multicenter follow-up study should be carried out to verify predictive value of serum FGF21 level for DR and STDR. Third, the generalizability of our results to other ethnic groups with type 2 diabetes remained elusive.

\section{CONCLUSIONS}

Serum FGF21 level was found to be positively associated with the severity of DR in Chinese patients with type 2 diabetes. Additionally, serum FGF21 level is a potential indicator of STDR. The serum FGF21 level $>554.69 \mathrm{pg} /$ $\mathrm{mL}$ indicates over eight-time enhancement of STDR occurrence.

Acknowledgements The authors would like to thank TopEdit (www.topeditsci. com) for the linguistic assistance provided during the preparation of this manuscript.

Contributors LH conceived the study. SJ designed the research. SJ and NX conducted the experiments. NX and SJ collected clinical data. NX and SJ analyzed the data. SJ wrote the manuscript. LH revised the manuscript. All authors approved this submission.

Funding This study was financially supported by Liaoning Natural Science Fund Plan Guidance Project (grant no. 2019-ZD-0774).

Competing interests None declared.

Patient consent for publication Not required.
Ethics approval The study was approved by the Human Research and Ethics Committee of the Fourth Affiliated Hospital of China Medical University and the Fourth People's Hospital of Shenyang, and was conducted in accordance with the Declaration of Helsinki. All the procedures were performed in accordance with the ethical standards of the institutional and/or national research committee and with the 1964 Declaration of Helsinki. This article does not contain any animal-based studies performed by none of the authors.

Provenance and peer review Not commissioned; externally peer reviewed.

Data availability statement Data are available on reasonable request.

Open access This is an open access article distributed in accordance with the Creative Commons Attribution Non Commercial (CC BY-NC 4.0) license, which permits others to distribute, remix, adapt, build upon this work non-commercially, and license their derivative works on different terms, provided the original work is properly cited, appropriate credit is given, any changes made indicated, and the use is non-commercial. See: http://creativecommons.org/licenses/by-nc/4.0/.

ORCID ID

Lingling Han http://orcid.org/0000-0002-1032-2842

\section{REFERENCES}

1 International Diabetes Federation (IDF). IDF diabetes atlas ninth edition, 2019. Available: https://diabetesatlas.org/en/resources/

2 Fong DS, Aiello L, Gardner TW, et al. Diabetic retinopathy. Diabetes Care 2003;26:226-9.

3 Liu L, Wu X, Liu L, et al. Prevalence of diabetic retinopathy in mainland China: a meta-analysis. PLoS One 2012;7:e45264.

4 Yang Q-H, Zhang Y, Zhang X-M, et al. Prevalence of diabetic retinopathy, proliferative diabetic retinopathy and non-proliferative diabetic retinopathy in Asian T2DM patients: a systematic review and meta-analysis. Int J Ophthalmol 2019;12:302-11.

5 American Diabetes Association. 11. Microvascular Complications and Foot Care: Standards of Medical Care in Diabetes-2019. Diabetes Care 2019;42:S124-38.

6 Strączkowski M, Karczewska-Kupczewska M, Adamska A, et al. Serum fibroblast growth factor 21 in human obesity: regulation by insulin infusion and relationship with glucose and lipid oxidation. Int $J$ Obes 2013;37:1386-90.

7 Gao R-Y, Hsu B-G, Wu D-A, et al. Serum fibroblast growth factor 21 levels are positively associated with metabolic syndrome in patients with type 2 diabetes. Int J Endocrinol 2019;2019:1-8.

8 Zhang W, Chu S, Ding W, et al. Serum level of fibroblast growth factor 21 is independently associated with acute myocardial infarction. PLoS One 2015;10:e0129791.

9 Zhang X, Hu Y, Zeng H, et al. Serum fibroblast growth factor 21 levels is associated with lower extremity atherosclerotic disease in Chinese female diabetic patients. Cardiovasc Diabetol 2015;14:32.

10 American Diabetes Association. Standards of medical care in diabetes-2012. Diabetes Care 2012;35 Suppl 1:S11-63.

11 Wilkinson CP, Ferris FL, Klein RE, et al. Proposed International clinical diabetic retinopathy and diabetic macular edema disease severity scales. Ophthalmology 2003;110:1677-82.

12 Zhang X, Yeung DCY, Karpisek M, et al. Serum FGF21 levels are increased in obesity and are independently associated with the metabolic syndrome in humans. Diabetes 2008;57:1246-53.

13 Chen C, Cheung BMY, Tso AWK, et al. High plasma level of fibroblast growth factor 21 is an independent predictor of type 2 diabetes: a 5.4-year population-based prospective study in Chinese subjects. Diabetes Care 2011;34:2113-5.

14 Knott ME, Minatta JN, Roulet L, et al. Circulating fibroblast growth factor 21 (Fgf21) as diagnostic and prognostic biomarker in renal cancer. J Mol Biomark Diagn 2016;1. doi:10.4172/2155-9929.S2015. [Epub ahead of print: 2005 2016].

15 Leske MC, Wu S-Y, Hennis A, et al. Hyperglycemia, blood pressure, and the 9-year incidence of diabetic retinopathy: the Barbados eye studies. Ophthalmology 2005;112:799-805.

16 Pang C, Jia L, Jiang S, et al. Determination of diabetic retinopathy prevalence and associated risk factors in Chinese diabetic and prediabetic subjects: Shanghai diabetic complications study. Diabetes Metab Res Rev 2012;28:276-83.

17 Lin Y, Xiao Y-cheng, Zhu H, et al. Serum fibroblast growth factor 21 levels are correlated with the severity of diabetic retinopathy. $J$ Diabetes Res 2014;2014:1-6. 
18 Mousavi Z, Bonakdaran S, Sahebkar A, et al. The relationship between serum levels of fibroblast growth factor 21 and diabetic retinopathy. Excli J 2017;16:1249-56.

19 Yaghoobi G, Shokoohi-Rad S, Jafarzadeh H, et al. Serum fibroblast growth factor 21 in patients with and without pterygia. J Ophthalmic Vis Res 2020;15:38-44.

20 Ong K-L, Januszewski AS, O'Connell R, et al. Relationship of fibroblast growth factor 21 with baseline and new on-study microvascular disease in the fenofibrate intervention and event lowering in diabetes study. Diabetologia 2015;58:2035-44.

21 Esteghamati A, Momeni A, Abdollahi A, et al. Serum fibroblast growth factor 21 concentrations in type 2 diabetic retinopathy patients. Ann Endocrinol 2016;77:586-92.

22 Jung $\mathrm{C}-\mathrm{H}$, Jung S-H, Kim B-Y, et al. The U-shaped relationship between fibroblast growth factor 21 and microvascular complication in type 2 diabetes mellitus. J Diabetes Complications 2017;31:134-40.

23 Yang M, Zhang L, Wang C, et al. Liraglutide increases FGF-21 activity and insulin sensitivity in high fat diet and adiponectin knockdown induced insulin resistance. PLoS One 2012;7:e48392.
24 Rusu CC, Racasan S, Kacso IM, et al. The metabolic hormone FGF21 is associated with endothelial dysfunction in hemodialysis patients. Int Urol Nephrol 2017;49:517-23.

25 Holland WL, Adams AC, Brozinick JT, et al. An FGF21-adiponectinceramide axis controls energy expenditure and insulin action in mice. Cell Metab 2013;17:790-7.

26 Lin Z, Tian H, Lam KSL, et al. Adiponectin mediates the metabolic effects of FGF21 on glucose homeostasis and insulin sensitivity in mice. Cell Metab 2013;17:779-89.

27 Lin Z, Pan X, Wu F, et al. Fibroblast growth factor 21 prevents atherosclerosis by suppression of hepatic sterol regulatory elementbinding protein-2 and induction of adiponectin in mice. Circulation 2015;131:1861-71.

28 Pan X, Shao Y, Wu F, et al. FGF21 Prevents Angiotensin II-Induced Hypertension and Vascular Dysfunction by Activation of ACE2/ Angiotensin-(1-7) Axis in Mice. Cell Metab 2018;27:1323-37.

29 Ying L, Li N, He Z, et al. Fibroblast growth factor 21 ameliorates diabetes-induced endothelial dysfunction in mouse aorta via activation of the CaMKK2/AMPK $\alpha$ signaling pathway. Cell Death Dis 2019;10:665. 